\title{
Helium Neutral Beam Injection into ASDEX Upgrade
}

\author{
C. Hopf, D. Rittich, J. Schäffler, J. Thalhammer, and the ASDEX Upgrade Team \\ Max-Planck-Institut für Plasmaphysik, Boltzmannstr. 2, 85748 Garching, Germany
}

\begin{abstract}
ASDEX Upgrade's (AUG) neutral beam injection (NBI) system is primarily designed for deuterium injection and delivers 20 MW heating power from two injectors with four beams each at 60 and $93 \mathrm{keV}$, respectively. As opposed to the cryo pumps of the JET NBI, the Ti getter pumps of the AUG NBI with a pumping speed of $3 \times 10^{6} \ell / \mathrm{s}$ for hydrogen do not pump helium at all, leaving only the conventional pumping system with $<6 \times 10^{3} \ell / \mathrm{s}$ for He. This imposes constraints on the possible operation in helium. In order to prepare for AUG He plasma campaigns, serious trials to operate the AUG NBI with He began in 2014. It was found that despite the lack of high speed pumping up to two beams per injector could be operated simultaneously at reduced feed gas flow without particular restrictions on the beam-on time. For injector 1 the power per beam was limited to $550 \mathrm{~kW}$ at $40 \mathrm{keV}$ by the required filament current in its arc sources, while for injector 2 the limitation came from the bending magnet's power supply that restricted the beam energy to $68 \mathrm{keV}$ and the NBI power to $730 \mathrm{~kW}$ per beam. Thus the maximum available NBI heating power in He amounts to $2.6 \mathrm{MW}$ for $10 \mathrm{~s}$, the maximum discharge duration of AUG. Helium neutral beam injection into plasma was first tried out in 2014 for two discharges. In 2015 a dedicated He campaign used He NBI in a total of 44 discharges. As He is almost not pumped in the injectors the neutral gas flow into the torus is comparable with the total He gas puff.
\end{abstract}

Keywords: Neutral beam injection, helium, ASDEX Upgrade

Published with Fusion Engineering and Design (Fus. Eng. Des. 123 (2017) 281-284)

\section{Introduction}

Neutral beam injection using helium instead of the hydrogen isotopes usually injected can be of interest for a variety of reasons. Firstly, when heating He discharges using $\mathrm{He}$ also for the neutral beams avoids dilution of the plasma with hydrogen. Secondly, ${ }^{4} \mathrm{He}$ injection can be used to simulate energetic alphas, e.g. to test alpha particle diagnostics for D-T reactors $[1,2]$ or study their slowing down [3]. Helium NBI was employed on a number of devices, most notably on JET with both ${ }^{3} \mathrm{He}$ and ${ }^{4} \mathrm{He}[4,5]$ and up to $16 \mathrm{MW}$ and on DIII-D with $6 \mathrm{MW}$ of ${ }^{4} \mathrm{He}[6]$.

A general difficulty with NBI is the pumping of the large feed gas flows into the NBI sources. For example, when operated in deuterium, between 25 and $40 \mathrm{mbar} \ell / \mathrm{s}$ are fed into each of the ASDEX Upgrade NBI sources and the same amount into each of the neutralizers. This amounts to $200-320 \mathrm{mbar} \ell / \mathrm{s}$ for a whole injector box when all of its four sources are operated. The requirement of keeping reionization losses of the neutralized beam low demands a pressure below $10^{-4} \mathrm{mbar}$ along the entire beamline. Hence, the required pumping speed is approx. $3 \times 10^{6} \ell / s$. Large cryo or getter pumps are used for this purpose. The NBI on AUG works with Ti sublimation getter pumps [7].

Both DIII-D and JET, whose NBI systems have cryo pumps, used argon frosting of the pumps to temporarily enable them

Email address: Christian.Hopf @ipp.mpg.de (C. Hopf, D. Rittich, J. Schäffler, J. Thalhammer, and the ASDEX Upgrade Team) to pump helium $[6,4]$. Using this technique the NBI systems could be operated without additional constraints on beam duration, while without argon frosting DIII-D reports a maximum beam-on time of $500 \mathrm{~ms}$ due to the pressure rise in the NBI box. With AUG NBI's Ti sublimation pumps this technique is not applicable and pure He operation had also not been envisaged at the time of its design.

So far traces of fast He neutrals could be produced on demand by admixing approx. $5 \%$ of He to the $\mathrm{D}_{2}$ feed gas of source number 3 that is especially equipped for this purpose. As the charge-to-mass ratio of ${ }^{4} \mathrm{He}^{+}$and $\mathrm{D}_{2}^{+}$are the same the residual singly-charged $\mathrm{He}$ ions after the neutralizer deposit their energy on the existing $\mathrm{D}_{2}^{+}$ion dump in this case. Pure He beam injection had never been seriously attempted and severe limitations regarding the number of beams that can be used simultaneously and the beam-on time had to be expected. In 2014 first tests with pure He beams began on one injector, followed by tests with the other injector in 2015. Still in 2015 a dedicated two-weeks He campaign used He NBI in 44 discharges.

In Section 2 we present the operational details and experience from an NBI standpoint while Section 3 discusses the experience from an application point of view.

\section{NBI operation in He}

The NBI system on ASDEX Upgrade features two injectors with four beams each that differ in injection geometry [8]. In standard deuterium operation each of the beams has a neutral 


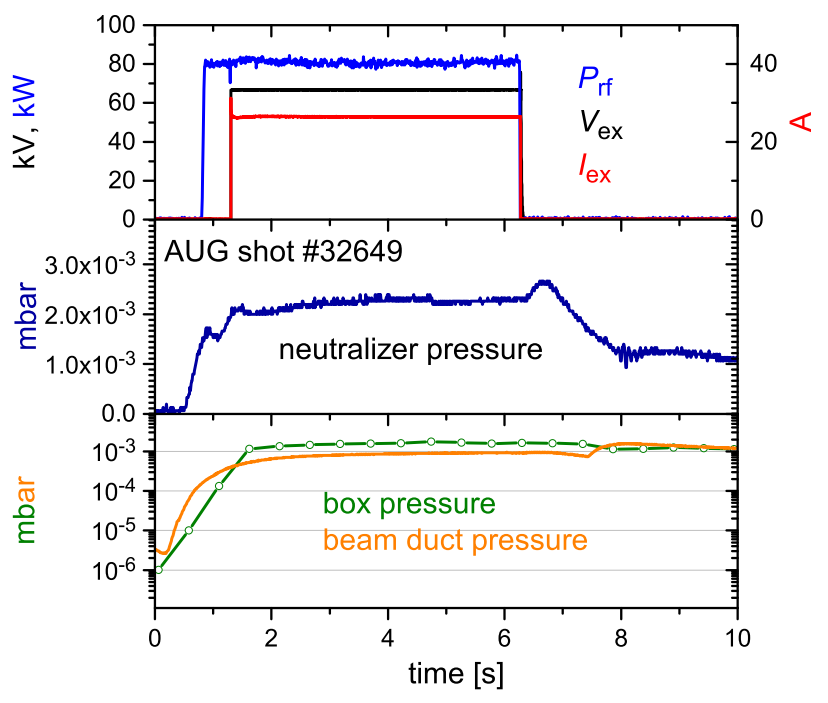

Figure 1: Time traces of some beamline parameters in operation with $\mathrm{He}$ as feed gas: rf power $P_{\mathrm{rf}}$, extraction voltage $V_{\mathrm{ex}}$, and extracted current $I_{\mathrm{ex}}$ of source number 8 , and the pressures measured in the neutralizer of beam source 8 , in the NBI box, and in the beam duct.

power of $2.5 \mathrm{MW}$. While injector 1 uses arc sources that are very similar to JET's PINIs and operates at $60 \mathrm{kV}$ full acceleration voltage, injector 2 has rf sources and a maximum acceleration voltage for deuterium of $93 \mathrm{kV}$.

Neutral beam accelerators have to be operated at an optimum perveance for which the beam's - or, more precisely, the beamlet's - divergence is minimal. For a single stage beam accelerator the perveance is defined as $I_{\mathrm{ex}} / V_{\mathrm{ex}}^{3 / 2}$, where $I_{\mathrm{ex}}$ is the extracted current and $V_{\mathrm{ex}}$ the extraction/acceleration voltage. For a given accelerator grid geometry this optimum perveance is proportional to $\sqrt{Z / M}$, where $Z$ and $M$ are the accelerated ion's charge state and mass number [9]. The fraction of doubly charged He ions in the beam is negligible due to the high second ionization energy of $\mathrm{He}$ of $54.4 \mathrm{eV}$. Accordingly, the perveance for the $\mathrm{He}^{+}$beams was chosen as the optimum perveance of the deuterium beams' full energy component (determined in perveance scans using beam emission spectroscopy) divided by $\sqrt{2}$.

As opposed to the aforementioned deuterium beams with helium admixture, the residual ions from the pure He beams cannot simply be deflected onto the $\mathrm{D}_{2}^{+}$dumps as these cannot take such high powers. Hence, the bending magnet current has to be increased by a factor of $\sqrt{2}$ for the He beams such that $\mathrm{He}^{+}$hits the main ion dumps.

The main anticipated problem was the lack of He pumping by the Ti getter pumps that might lead to high background pressure and consequently large reionization losses. Therefore we operated the sources with the minimum possible flow of $\mathrm{He}$ at which they were found to run properly and without additional gas feed to the neutralizer. Thus the gas flow was $\approx 40 \mathrm{mbar} \ell / \mathrm{s}$ per arc source of injector 1 and $\approx 25 \mathrm{mbar} \ell / \mathrm{s}$ per rf source of injector 2 . It is worth noting that with these settings and $\approx 30 \mathrm{~A}$ extracted current between 17 and $30 \%$ of the feed gas is converted into extracted ions. Initially we operated only one source per box and for short times of around $100 \mathrm{~ms}$ in order to watch carefully for any alarming temperature readings of the thermocouples along the beamline and to keep the total amount of introduced gas low. However, the gas inlet starts already $1 \mathrm{~s}$ before the beam pulse as can be seen from the time lag between the rise of the neutralizer pressure and the switching on of the extraction voltage in Fig. 1. Gradually the beam time was extended and it was finally found that even a second beam per box could be added without any time restrictions. In such shots the background $\mathrm{He}$ gas pressure reaches a steady value of $\approx 1 \times 10^{-3}$ mbar while the pressure at the beam duct exit in the torus midplane is $\approx 3 \times 10^{-5}$ mbar. Assuming a linear pressure drop between those values along the $3.4 \mathrm{~m}$ between the calorimeter (the place of the power measurement) and the duct exit and assuming $300 \mathrm{~K}$ as the gas temperature this would lead to reionization losses of $\approx 27 \%$. A higher background gas temperature due to beam-induced heating would reduce these losses, e.g. for $1000 \mathrm{~K}$ to $9 \%$. Given their large uncertainty, reionization losses are not accounted for in the remainder of the paper. Generally, there is a trade off between the reduction of the reionization losses by reducing the source and neutralizer gas and the achievable neutralization efficiency, that is negatively affected when reducing the neutralizer pressure. As we will show below, the latter effect is not very pronounced in our case.

The rf sources of injector 2 proved entirely unproblematic when operated in He. The fast feedback control of the extracted current using the rf power as actuator ensured operation at the preset perveance without the need for any tuning. The extraction voltage was ultimately limited to $72.6 \mathrm{kV}$ in shots onto the calorimeter without an AUG plasma discharge by the power supply of the bending magnet. With the pre-calculated optimum perveance of $1.58 \times 10^{-6} \mathrm{~A} / \mathrm{V}^{3 / 2}$ the corresponding extracted current was $30.7 \mathrm{~A}$. In He $95 \mathrm{~kW}$ of rf power were needed while for the usual deuterium beams at $93 \mathrm{kV} / 65 \mathrm{~A}$ only about $90 \mathrm{~kW}$ are required. Operating the beams in ASDEX Upgrade discharges we found that the bending magnet power supply had an even lower maximum current due to a slightly lower primary voltage when more consumers take power from the grid. This reduced the maximum beam energy further to $68 \mathrm{keV}$ at $26 \mathrm{~A}$. In the arc sources of injector 1 the expected optimum perveance was $3.60 \times 10^{-6} \mathrm{~A} / \mathrm{V}^{3 / 2}$. In order to achieve the appropriate extracted current the arc current had to be adjusted. The operating parameters were finally limited to $40 \mathrm{kV}$ and $30 \mathrm{~A}$ extracted current as with $15 \mathrm{~A}$ the required current in the arc source filaments was already exceeding that in full power $\mathrm{D}$ or $\mathrm{H}$ operation and there was a risk that increasing it further might seriously reduce the filaments' life time. As opposed to injector 2 this of course is not a hard limit. It should be mentioned here, that despite the higher neutral beam power in He the JET PINIs operated at lower extracted currents.

For the beams of injector 2 the injected neutral beam power deposited on the actively cooled panels of the calorimeter was calculated by $P_{\text {cal }}=\Phi \cdot c \cdot t_{\mathrm{on}} \int\left(T_{\mathrm{RL}}(t)-T_{\mathrm{FL}}(t)\right) \mathrm{d} t$, where $T_{\mathrm{FL}}$ and $T_{\mathrm{RL}}$ are the temperatures in the feed and return lines of the calorimeter's high pressure water cooling circuit, $\Phi$ is the 


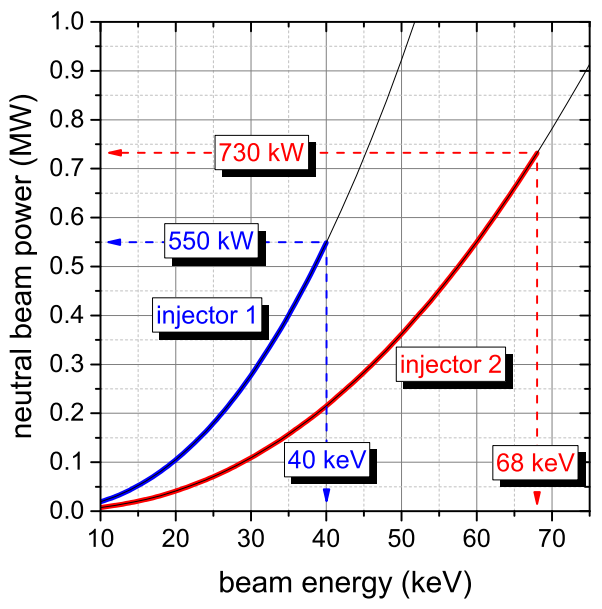

Figure 2: Estimated neutral beam power per beam as function of beam energy. The thick colored lines indicate the accessible range, the thin lines are extrapolations beyond the currently possible extraction voltage for $\mathrm{He}$.

cooling water mass flow, $c$ is the heat capacity of water and $t_{\text {on }}$ is the beam-on time. At $72.6 \mathrm{kV}$ acceleration voltage this power was $973 \mathrm{~kW}$.

The neutralization efficiency $F_{0}$ was determined by comparing two He beams of the same energy fired onto the calorimeter; in the first case the bending magnet was switched on as usual, deflecting the non-neutralized ions into the residual ion dump; in the second case the magnet stayed off, admitting both the neutrals and the residual ions to strike the calorimeter. This yielded $F_{0}=0.57$ which is $75 \%$ of the maximum neutralization efficiency $F_{0 \infty}=0.76$ [10] at this energy despite our minimized gas flow into source and neutralizer.

The neutral beam power as a function of extraction voltage was extrapolated from this value using the relationship $P_{\text {ex }}(V)=V I=\mathcal{P} V^{5 / 2}$ for the extracted beam power, where $\mathcal{P}=I / V^{3 / 2}$ is the perveance. The neutral beam power can then be estimated by

$$
P_{\mathrm{NBI}}=s T_{\mathrm{duct}} F_{0 \infty}(E) P_{\mathrm{ex}}(V)
$$

where $T_{\text {duct }}=0.86$ is the estimated geometric transmission of the NBI duct and $s$ is a scaling factor $<1$ that describes all losses that are otherwise not accounted for and is assumed to be independent of beam energy. That latter assumption also implies that the actual neutralization efficiency $F_{0}$ is assumed to scale with energy like the equilibrium fraction $F_{0 \infty}(E)$. By equating $s F_{0 \infty} P_{\text {ex }}$ with the power measured at the calorimeter for the $72.6 \mathrm{keV}$ neutral beam the value of $s$ was determined to be 0.57 . For plasma heating from injector 2 with the maximally possible $68 \mathrm{keV}$, as explained earlier, we thus obtain $732 \mathrm{~kW}$ neutral beam power.

For the beams of injector 1 it was assumed that Eq. (1) applies with the same $s$ as for injector 2. Then only the perveance is different for the two injectors. The estimated neutral beam powers as a function of beam energy are plotted in Fig. 2.
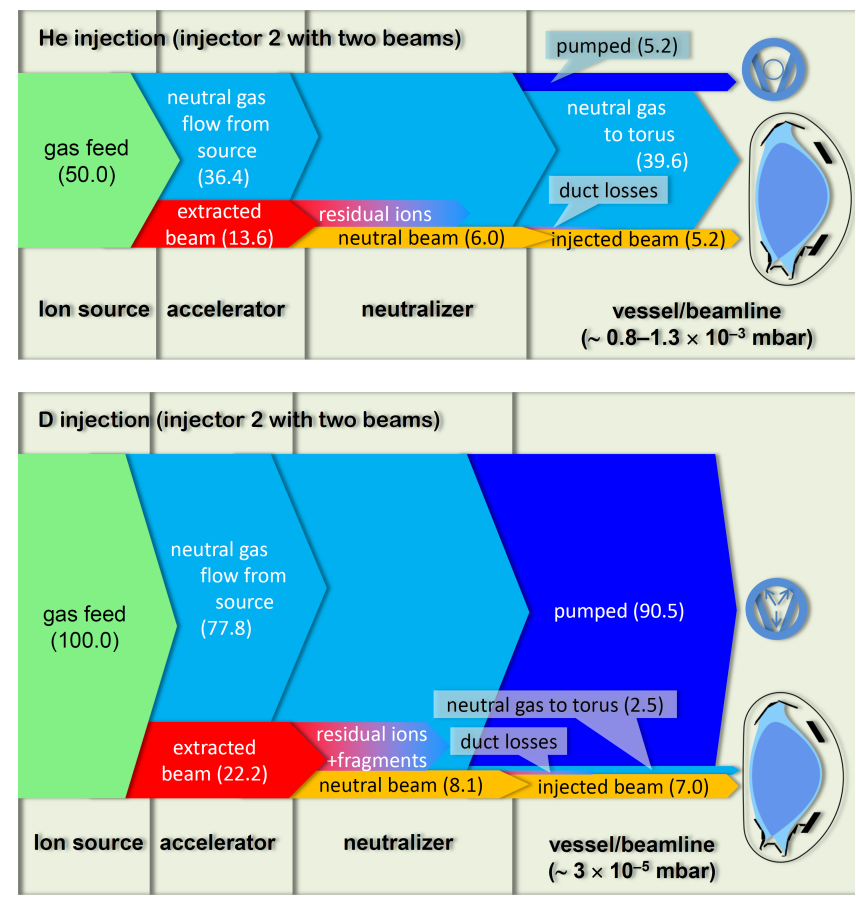

Figure 3: Gas balance in steady state for operation of injector 2 with 2 helium beams (top) and two deuterium beams (bottom). All numbers without explicitly given dimensions are in $m b a r \ell / s \mathrm{He}$ and $\mathrm{D}_{2}$ respectively.

\section{Plasma response}

Due to the lack of He pumping in the NBI boxes He NBI contributes significantly to plasma fuelling as we will show taking AUG shot \#32648 as an example. The shot was fuelled with $\approx 3.5 \times 10^{21} \mathrm{He}$ atoms via gas puffing through a torus gas valve. It had two beams on for $5 \mathrm{~s}$ and the sources were fed by a gas flow of $25 \mathrm{mbar} \ell / \mathrm{s}$ each that was on for 6 seconds. That converts to $7.2 \times 10^{21}$ atoms. Of these, $0.7 \times 10^{21}$ atoms were directly shot into the plasma as neutral beam, leaving $6.5 \times 10^{21}$ atoms as neutral gas in the box. As the pressure in the NBI box was about $8.7 \times 10^{-4}$ mbar, the turbo pumps with their pumping speed of $\approx 6 \times 10^{3} \mathrm{l} / \mathrm{s}$ must have pumped approximately only $0.8 \times 10^{21}$ atoms. The remaining $5.7 \times 10^{21}$ atoms are expected to reach the plasma as neutral gas. This shows that the He introduced by NBI is almost twice as much as that introduced by gas puffing, which was tolerable for the specific scenario but could pose a problem for other types of He discharges. The situation is distinctly different from most cases in deuterium operation, where D fuelling by NBI is rather negligible.

Figure 3 visualizes the gas balance in steady state for He NBI with two beams and for $\mathrm{D}_{2}$ operation in comparison. For $\mathrm{He}$ operation the flow transmitted through the duct was calculated as the feed gas flow that is not converted into injected beam nor pumped by the turbo pumps of known pumping speed. For $\mathrm{D}_{2}$ operation the pumping speed of the Ti getter pumps is not precisely known. Therefore the neutral gas flow through the duct was estimated assuming the same conductance of the duct that can be calculated from box pressure and neutral duct gas flow in the He case. The pumped flow for $\mathrm{D}_{2}$ is then given by the rest that is not injected beam. Thus, the Ti getter pumping 


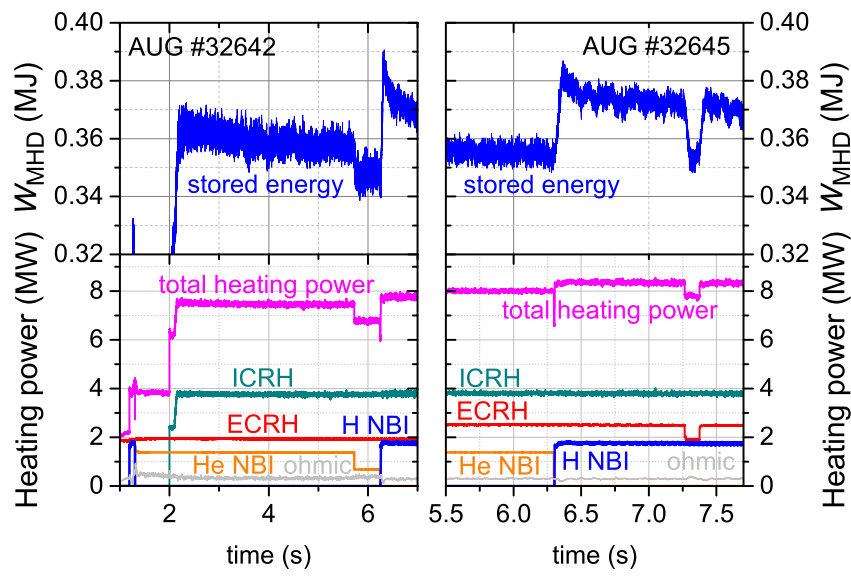

Figure 4: Time traces of the stored energy, the heating powers of $\mathrm{He} / \mathrm{D}_{2} \mathrm{NBI}$, ohmic heating, ECRH, ICRH as well as the total heating power for two AUG discharges.

speed can be estimated a posteriori, yielding $2.8 \times 10^{6} \ell / \mathrm{s}$ in good agreement with their theoretical value.

Figure 3 demonstrates that, as opposed to deuterium (or hydrogen) operation, He operation completely relies on pumping of the NBI box through the duct in order to achieve a low enough steady background pressure. Such a steady state cannot be reached in shots with the duct closed by the torus valve and the calorimeter lowered, as then pumping is dominantly volume pumping by the $\approx 30 \mathrm{~m}^{3}$ of the NBI box.

Figure 4 shows the response of the plasma stored energy $W_{\text {MHD }}$ to changes of the heating power. In discharge \#32642 the He NBI power drops around $5.7 \mathrm{~s}$ from nominally 1.37 to $0.68 \mathrm{MW}$ as one beam trips, and this drop is clearly visible in the stored energy. In discharge \#32645 the response of $W_{\mathrm{MHD}}$ to the switch from 1.37 MW He NBI to $1.78 \mathrm{MW} \mathrm{H} \mathrm{NBI}$ at $6.3 \mathrm{~s}$ is of very similar magnitude as the response to a temporary drop of ECRH power by $690 \mathrm{~kW}$. This might be an indication that either the He NBI power is slightly overestimated, e. g. by neglecting reionization losses, or could be related to the different characteristics of He NBI and ECR heating.

Figure 5 shows the radial, flux-surface-integrated heating power profiles calculated with TRANSP for discharge \#32664. The solid lines correspond to a time point when two He beams at $66 \mathrm{keV}$ are the only NBI, and the dashed lines to a time when only one $\mathrm{H}$ beam at $53 \mathrm{keV}$ is on. Electron temperature and density profiles are almost identical for the two time points. It is well visible that, despite the He beams' higher energy, the ion-to-electron heating ratio is higher for $\mathrm{He}$ at all radii. The hydrogen beam deposits a much higher fraction of its power outside $\rho_{\text {tor }}=0.6$.

\section{Summary}

We have successfully demonstrated helium neutral beam injection on ASDEX Upgrade and applied it in 44 discharges during the 2015 helium campaign. Helium NBI is possible with a pulse length up to the maximum AUG discharge duration of

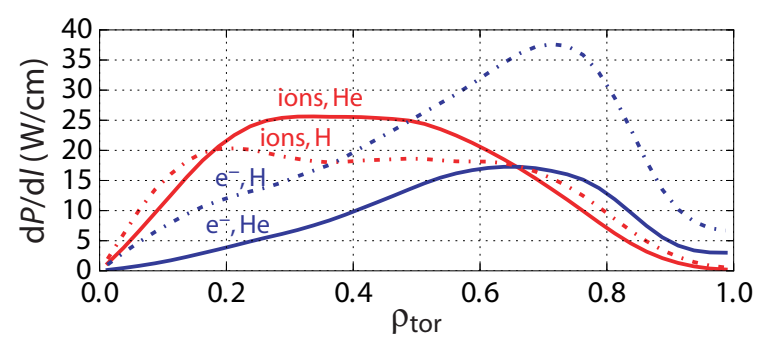

Figure 5: Flux-surface-integrated, radial heating power density profiles of the neutral beams in discharge \#32664. Blue lines show heating power to the electrons, red lines heating power to the ions. The solid lines refer to a time point when two He beams were the only NBI, the dashed lines to a time point when one $\mathrm{H}$ beam was the only NBI.

$10 \mathrm{~s}$ with up to two beams per injector. In the absence of high speed pumps for He in the NBI boxes, the long pulses rely entirely on the effective pumping through the beam duct to the torus. The downside of this situation are high gas fuelling of the AUG discharges from the NBI boxes that typically exceeded the amount of He puffed through the torus valves by a factor of two already if only two He beams were used from one box.

The maximum extracted beam current on injector 1 was limited by the filament current of its arc sources. That restricted the extraction voltage to $40 \mathrm{kV}$ and the beam power to $\approx 550 \mathrm{~kW}$. Injector 2 was limited to $68 \mathrm{kV}$ and $\approx 730 \mathrm{~kW}$ by the maximum bending magnet current. These estimated powers do not account for reionization losses in the beam duct that were estimated to be somewhere between $\sim 10$ and $\sim 30 \%$.

\section{Acknowledgement}

This work has been carried out within the framework of the EUROfusion Consortium and has received funding from the Euratom research and training programme 2014-2018 under grant agreement No 633053. The views and opinions expressed herein do not necessarily reflect those of the European Commission.

[1] A. Kappatou, E. Delabie, R. J. E. Jaspers, and M. G. von Hellermann, Nucl. Fusion 52, 043007 (2012).

[2] A. Kappatou, Ph.D. thesis, TU Eindhoven (2014).

[3] M. G. von Hellermann, J. F. W. G. F. Coret, L. D. Horton, R. W. T. König, W. Mandl, and H. P. Summers, Plasma Phys 35, 799 (1993).

[4] F. B. Marcus, J. M. Adams, D. V. Bartlett, V. Bhatnagar, J. Bickley, M. Bures, D. J. Campbell, C. D. Challis, J. Christiansen, S. Conroy, et al., Plasma Phys. Control. Fusion 34, 1371 (1992).

[5] D. C. McDonald, J. G. Cordey, E. Righi, F. Ryter, G. Saibene, R. Sartori, B. Alper, M. Becoulet, J. Brzozowski, I. Coffey, et al., Plasma Phys. Control. Fusion 46, 519 (2004).

[6] J. C. Phillips, D. H. Kellman, R. Hong, J. Kim, and G. M. Laughon, Proceedings of the 16th IEEE/NPSS Symposium Fusion Engineering p. 852 (1995)

[7] G. Orozco, M. Froeschle, B. Heinemann, C. Hopf, R. Nocentini, R. Riedl, and A. Staebler, Fusion Eng. Des. 89, 3070 (2014).

[8] B. Streibl, P.-T. Lang, F. Leuterer, J.-M. Noterdaeme, and A. Stäbler, Fusion Sci. Technol. 44, 578 (2003).

[9] E. Speth, M. Ciric, J. H. Feist, P. Frank, B. Heinemann, W. Kraus, F. Probst, R. Riedl, R. Trainham, O. Vollmer, et al., Fusion Eng. Des. 46, 383 (1999).

[10] S. K. Allison, Rev. Mod. Phys. 30, 1137 (1958). 